\title{
CovID-19 COVID-19 pandemic personal development plan: A model for maximising higher specialty training
}

\author{
Authors: Jatinder S Minhas, ${ }^{A}$ Daniel Pan, ${ }^{B}$ Shirley Sze, ${ }^{C}$ Manish Pareek ${ }^{D}$ and Chris ] Miller ${ }^{E}$
}

\begin{abstract}
The coronavirus disease 2019 (COVID-19) pandemic has led to multiple service delivery changes across acute care sectors in the UK. Due to increased responsibility for care of COVID-19 patients, medical trainees across all specialties might experience difficulty in achieving certain competencies for their training curriculum due to changes in learning opportunities. While there might be a tendency to perceive these changes negatively in terms of the impact on training, we think this unprecedented situation might present a unique learning opportunity. A group of geriatric medicine trainees and trainers devised an innovative, forward-thinking specific training plan based on existing Joint Royal Colleges of Physicians Training Board geriatric medicine curricula, encouraging development of a personal development plan (PDP) tailored to the pandemic. This model could be considered for all specialty training curricula, providing a proactive approach to optimising training during the pandemic. By formulating a 'pandemic PDP' early and considering methods to maximise learning, training needs can be met even in these extraordinary times.
\end{abstract}

KEYWORDS: COVID-19, training, medical education

DOI: $10.7861 /$ fhj.2020-0057

\section{Introduction}

The coronavirus disease 2019 (COVID-19) pandemic caused by the severe acute respiratory syndrome coronavirus 2 (SARSCoV-2) virus has led to multiple service delivery changes across primary and secondary care sectors. The UK Government stated early during the pandemic that that those aged 70 years and older are at an increased risk of severe illness from COVID-19.

Authors: Aspecialist registrar, geriatric medicine, Leicester Royal Infirmary, Leicester, UK and University of Leicester, Leicester, UK; ${ }^{B}$ specialist registrar, infectious diseases, Leicester Royal Infirmary, Leicester, UK; ' Cacademic clinical fellow, University of Leicester, Leicester, UK; 'D consultant, infectious diseases, Leicester Royal Infirmary, Leicester, UK; ${ }^{\mathrm{E}}$ Consultant, geriatric medicine, Leicester Royal Infirmary, Leicester, UK
In the UK, geriatric medicine (GM) training is delivered by 1,223 consultants to 1,010 specialist registrars (SpRs) (both training and non-training grades). ${ }^{2}$ The vast majority of GM SpRs are based in acute hospitals and training is coordinated through and in line with the Joint Royal Colleges of Physicians Training Board (JRCPTB) Geriatric Medicine Curriculum (Updated 2016). ${ }^{3}$ On 16 March 2020, the JRCPTB released a statement commenting on the pandemic: ${ }^{4}$

The pandemic of COVID-19 will lead to changes in learning opportunities for trainees in Core Medical, Internal Medicine and Medical Specialty training both in terms of experiences and in assessments. Their ability to complete rotations in specific elements of training may be compromised and, at a time when clinical teams are likely to be stretched significantly, opportunities for specific supervised learning events (SLES) may be limited.

While for many specialties this may hold true, the rapid progression of the pandemic and increased responsibility for the care of COVID-19 patients by GM teams has taught us some lessons. Specifically, there are training opportunities in all settings, from the front door (acute frailty in emergency department) to the community hospital and care home. Managing the severity seen in our common GM 'giants', including delirium, is proving challenging and atypical presentations predominate. ${ }^{5}$ Sadly, infection prevention procedures restrict the usual management approaches to several common presentations. Advanced care planning has been challenging for primary and secondary care clinicians alike. The National Institute for Health and Care Excellence (NICE) has encouraged the use of the clinical frailty scale (CFS) as part of a holistic assessment of patients admitted to hospital. ${ }^{6}$

The challenging clinical presentations seen require careful consideration of the efficacy and appropriateness of current management approaches and, importantly, link to established GM curriculum competencies, amplifying specific aspects of the training curriculum.

Challenges to delivering higher specialty training during the COVID-19 pandemic

During the COVID-19 pandemic, trainees across all specialties and levels of seniority will be experiencing different challenges. Some of these will occur within their speciality; for others, new challenges beyond the scope of their usual competencies and skills will be experienced. While there may be a tendency to perceive 
Table 1. COVID-19-specific learning opportunities aligned with the geriatric medicine higher specialty training curriculum ${ }^{3}$

$\begin{array}{ll}\begin{array}{l}\text { Common } \\ \text { competencies }\end{array} & \begin{array}{l}\text { CBDi-CEX } \\ \end{array} \\ & \\ & \\ & \\ \text { Comprehensive } & \text { Mini-CEX } \\ \text { geriatric } & \text { CBD } \\ \text { assessment } & \text { ACAT }\end{array}$

$\begin{array}{ll}\text { Diagnosis and } & \text { Mini-CEX } \\ \text { management of } & \text { CBD } \\ \text { acute illness } & \text { ACAT }\end{array}$

$\begin{array}{ll}\text { Planning } & \text { Mini-CEX } \\ \text { transfers of } & \text { CBD } \\ \text { care, including } & \text { ACAT } \\ \text { discharge } & \text { MSF }\end{array}$

$\begin{array}{ll}\text { Delirium } & \begin{array}{l}\text { Mini-CEX } \\ \text { CBD }\end{array} \\ \text { Dementia } & \begin{array}{l}\text { Mini-CEX } \\ \text { CBD }\end{array} \\ & \text { Mini-CEX } \\ \text { Continence, } & \text { CBD } \\ \text { falls, poor } \\ \text { mobility }\end{array}$

Infection control: PPE, minimising MDT exposure through joint working and contribution to nursing and HCA daily tasks during ward round

Breaking bad news: utilising advanced communication skills to triangulate clinical plans with family members, discuss advanced care planning and provide communication in last days (via telephone, video media etc)

Principles of medical ethics and confidentiality: managing lines of communication with families, best interest decision making regarding respiratory support specifically and de-escalation of care where appropriate (specifically NIV/CPAP)

Ethical research: engaging with recruiting COVID-19 trial research primary investigators and research nurses (eg RECOVERY trial)

Teaching and training: utilising opportunities for presenting cases and learning experiences with infectious diseases colleagues (joint MDT opportunities) and taking a lead in supporting learning for IMT and FY colleagues

Factors influencing health status of older people: daily appraisal of clinical progress in COVID-19positive patients

Be aware of and recognise age discrimination within healthcare systems: process of advocacy on admission, during stay and on discharge

Diagnostic skills in the context of complex multi-system pathologies: COVID-19 causes multiorgan dysfunction and the 'cytokine storm' requires early recognition and strong supportive care Functional status evaluation: accuracy is imperative and careful collateral to ensure accuracy of CFS is advised

Collaborative working: mandatory at all times, MDT approach leads to joint up COVID-19 working, helping to minimise premature discharge and improve transfers of care

Management of older patients in critical care units: both prior to ITU step-up and step-down, there are unique opportunities to support critical care colleagues

Secondary complications of acute illness in older people: COVID-19 associates with coagulopathy, secondary sepsis, complications of immobility and a host of other downstream sequelae. If complications occur, robust advanced care planning is needed

To communicate empathetically with carers of a seriously ill older patient regarding prognosis: regular exposure to this competency and often requires multiple conversations, opportunities for SpRs and consultants to engage at intervals with families and debrief

Diagnostic uncertainty: COVID-19 increases the risk of sudden death. Managing, communicating and dealing with this uncertainty is an important skill to develop

Variety of resources available following discharge: in the early COVID-19 pandemic period, these resources were limited, evolving and a clear understanding of infection risk, isolation procedures and avenues to support patients and families improves

Planning skills: resource allocation can be stretched, therefore early planning and acknowledgement for improvement can facilitate timely discharge

MDT planning meetings: these are regular and primarily about function and discharge planning. Additionally, they help ensure $\mathrm{OOH}$ nursing teams are aware of treatment plans

COVID-19 causes delirium, which can be severe, and infection prevention procedures restrict usual management approaches

Supporting COVID-19 patients with dementia presents unique challenges, particularly around ensuring personalised care, reassuring relatives and discharge planning

These domains are all associated with greater challenges in a COVID-positive cohort ward; constipation, higher risk of falls and greater associated dependency (due to limited exertional exercise tolerances) are common examples of the specific relevance

Nutritional assessment tool exposure and consideration for daily food and fluid charts is helpful to monitor trends in treatment response. Remote liaison with dietetics presents challenges and requires greater clarity on specific indications for input 
Table 1. COVID-19-specific learning opportunities aligned with the geriatric medicine higher specialty training curriculum ${ }^{3}$ (Continued)

\begin{tabular}{|c|c|c|}
\hline \multirow[t]{3}{*}{ Homeostasis } & $\begin{array}{l}\text { Mini-CEX } \\
\text { CBD }\end{array}$ & $\begin{array}{l}\text { Changes in fluid and electrolyte homeostasis and thermoregulation: highly relevant daily, } \\
\text { necessitates consideration for insensible losses (regular and persistent pyrexia a challenge) }\end{array}$ \\
\hline & & $\begin{array}{l}\text { Acid-base abnormalities: greater necessity for sampling both for respiratory and sepsis indications } \\
\text { - interpreting anion gaps, explanations for metabolic acidosis and careful escalation of T1 and T2 } \\
\text { respiratory failure are crucial skills to demonstrate }\end{array}$ \\
\hline & & $\begin{array}{l}\text { Ability to manage and lead cardiac arrest: both full and peri-arrest scenarios, consideration for PPE } \\
\text { and protecting team as COVID-19 increases the risk of a cardiac arrest and a worse outcome is } \\
\text { more likely }\end{array}$ \\
\hline \multirow[t]{3}{*}{ Palliative care } & $\begin{array}{l}\text { Mini-CEX } \\
\text { CBD }\end{array}$ & $\begin{array}{l}\text { Complex and unpredictable symptom profiles, widened appreciation for available agents needed } \\
\text { (AKI predominates in COVID-19, therefore greater understanding of renal and non-renal excreted } \\
\text { agents is helpful) }\end{array}$ \\
\hline & & $\begin{array}{l}\text { Bereavement care has logistical challenges and requires senior MDT members to support nursing } \\
\text { staff, in particular when notifyingrelatives }\end{array}$ \\
\hline & & $\begin{array}{l}\text { Appreciation for personal, cultural and religious background: liaison via various approaches with } \\
\text { hospital chaplaincy team for spiritual care }\end{array}$ \\
\hline
\end{tabular}

This list is far from exhaustive but designed to encourage engagement and innovation. This approach should be supported with a pandemic personal development plan. CFS = clinical frailty scale; CPAP = continuous positive airway pressure; $\mathrm{HCA}=$ healthcare assistant; $\mathrm{MDT}=$ multidisciplinary team; NIV = non-invasive ventilation; $\mathrm{OOH}=$ out-of-hours; $\mathrm{PPE}=$ personal protective equipment.

these challenges negatively in terms of their impact on training, this unprecedented situation does present a unique opportunity for trainees currently in GM higher speciality training (HST).

The GM curriculum differs from other medical specialties in that there are no procedural competencies and outpatient-based subspecialty curriculum elements are often supplemented by inpatient exposure (with continence, movement disorders and psychiatry of old age, for example). However, a commonality across all specialties is the presence of emergency rotas, service pressures and associated difficulties in obtaining SLEs and workplace-based assessments (WPBAs). Our early experiences caring for COVID-19-positive patients has highlighted numerous important learning experiences that trainees could use to further their personal development. These align with curriculum competencies and arguably offer opportunities to obtain greater evidence of achievement.

\section{Pandemic personal development plan}

Across the UK, GM departments are providing core support in acute hospitals during this COVID-19 pandemic. Trainers and trainees are at the heart of this. By being innovative, forward thinking and positive, training opportunities can be maximised. The GMspecific training plan shown in Table 1, devised by a GM trainer and trainee team with wider input from local trainers and trainees, provides a unique example of how all specialities can optimise training through encouragement of experiential learning linked to a pandemic personal development plan (PDP). Prior literature has demonstrated that formal development programs in circumstances involving a changing training landscape permit greater flexibility and responsiveness to challenges. ${ }^{7}$ COVID-19 patients have clinical presentations closely linking to many of the GM competencies, providing clear opportunities for training benefit; equally, some GM presentations are less obviously related and therefore clinical exposure will be less easily gained during the pandemic period. Through careful documentation of COVID-19 phenotypes encountered in the ward setting, described during cross-specialty meetings and outlined in the medical literature, the opportunity to link COVID-19 care to existing GM curriculum elements was deemed necessary.

Despite a paucity of literature currently existing to support approaches to caring for older people with COVID-19, we have explored current understanding to help support the themes raised in this training plan. We know screening and streaming of frail older people to COVID-specific facilities can be challenging. ${ }^{5}$ In a French study of older people, fever was the most common symptom. ${ }^{8}$ However, cough, delirium, dyspnoea and fatigue were also common. Additionally, lymphopenia, renal and hepatic impairment were common. Atypical signs of COVID-19 infection such as confusion, fall or diarrhoea were hypothesised to be associated with greater likelihood of severe infection. ${ }^{\text {? }}$ Furthermore, severity assessed using rapid scoring systems is more sensitive and specific in those $<65$ years of age than $>65$ years of age. ${ }^{9}$ From a sub-specialty perspective, the long-term consequences of this pandemic on Parkinson's disease (PD) is highly uncertain, and timely education and reassurance of patients may help ease the burden. ${ }^{10}$ Beyond the disease itself, several challenges have been identified during the pandemic which are likely to affect older people, including reductions in physical activity and corresponding increases in sedentary behaviours, increase in stress levels, nutritional challenges, and limited access to routine health care and/or home care visits.1 The aforementioned clinical presentations and complications of COVID-19 infection highlight the breadth of training opportunities for all medical specialties and in particular for those dual training in general internal medicine (GIM).

Interestingly, in the USA, there has been some negativity around the potential effects on training in GM, with a trainer stating:12

I worry about permanent effects of this pandemic on doctoring in general and geriatrics in particular, both because our bedside care has changed and because we hear public pronouncements denigrating the value of elderly lives. 
While this may hold true in other countries, our experience is that attempting to deliver care as we always do, while paying particular attention to COVID-19-specific elements, ensures training opportunities are maintained and potentially strengthened. As a Trust that recently adopted the 'Recommended Summary Plan for Emergency Care and Treatment' (ReSPECT) form process, enhanced learning and greater exposure to careful advanced care planning has been possible. This process is crucial as it ensures the patient's 'personal preference sits alongside the expectations and limits decided by their clinician'.13 Additionally, interaction and bidirectional learning between infectious diseases and GM teams has helped reinforce current approaches to caring for patients with COVID-19. Specifically, early during the pandemic, cross-specialty debriefs gave junior and senior colleagues an opportunity to share good practice and discuss evolving issues. In the event of a 'second wave' of the pandemic in the UK, a trainer and trainee meeting in the first week to develop a pandemic PDP could be encouraged, together with the establishment of weekly cross-specialty debriefs. This approach is of potential value to all specialties, with a pandemic PDP giving an opportunity for trainees to enter into a dialogue with trainers about the implications of COVID-19 clinical presentations on their specialty-specific JRCPTB curriculum competencies and to acknowledge how clinical exposure is likely to be affected (such as an expected reduction in specific non-emergency procedures in gastroenterology or cardiology). Future work in this area could involve assessing the relative performance of trainees who did/did not use this tool.

\section{Conclusion}

In conclusion, despite the challenges the pandemic has presented, GM training has evolved to incorporate greater training on infection management as well as greater exposure to many key curriculum areas. By acknowledging these early, formulating a pandemic PDP and considering individually how trainers and trainees can maximise training, progress can be made. GM training in UK is varied and comprehensive, and irrespective of the complexities that COVID-19 presents, GM trainers and trainees have skill sets that can help ensure a safe and supportive environment for those in their care.

\section{Acknowledgements}

The authors would like to thank Prof Tahir Masud and Prof Rowan Harwood for their kind contribution to the COVID specific training plan. The authors would also like to thank the British Geriatrics Society for their kind support.

\section{Conflicts of interest}

Jatinder S Minhas is supported by the National Institute for Health Research (NIHR clinical lectureship in older people and complex health needs). Manish Pareek is supported by the National Institute for Health Research (NIHR post-doctoral fellowship). The views expressed in this publication are those of the authors and not necessarily those of the NHS, the National Institute for Health Research or the Department of Health and Social Care.

\section{References}

1 Osama T, Pankhania B, Majeed A. Protecting older people from COVID-19: should the United Kingdom start at age 60? J $R$ Soc Med 2020;113:169-70.

2 NHS Digital. Data and information. https://digital.nhs.uk/data-andinformation [Accessed 24 April 2020].

3 Joint Royal Colleges of Physicians Training Board. Geriatric medicine. www.jrcptb.org.uk/specialties/geriatric-medicine [Accessed 24 April 2020].

4 Joint Royal Colleges of Physicians Training Board. COVID-19 and recognition of trainee progression in 2020. www.jrcptb.org.uk/news/ covid-19-and-recognition-trainee-progression-2020 [Accessed 24 April 2020].

5 Tay HS, Harwood R. Atypical presentation of COVID-19 in a frail older person. Age Ageing 2020;9:523-4.

6 National Institute of Health and Care Excellence. COVID-19 rapid guideline: critical care in adults. NICE guideline [NG159]. NICE, 2020. Available from www.nice.org.uk/guidance/NG159 [Accessed 24 April 2020]

7 Crampton M, Wilkinson D. The professional development program of the australian college of rural and remote medicine. Aust Fam Physician 2002;31:952-6.

8 Godaert L, Proye E, Demoustier-Tampere D et al. Clinical characteristics of older patients: The experience of a geriatric short-stay unit dedicated to patients with COVID-19 in France. J Infect 2020;81:e93-4.

$9 \mathrm{Hu} \mathrm{H}$, Yao N, Qiu Y. Comparing rapid scoring systems in mortality prediction of critical ill patients with novel coronavirus disease. Acad Emerg Med 2020;27:461-8.

10 Prasad S, Holla VV, Neeraja K et al. Parkinson's disease and COVID19: Perceptions and implications in patients and caregivers. Mov Disord 2020;35:912-4.

11 Schrack JA, Wanigatunga AA, Juraschek SP. After the COVID-19 pandemic: the next wave of health challenges for older adults. J Gerontol A Biol Sci Med Sci 2020 in press (doi: 10.1093/gerona/ glaa102).

12 Siegler EL. Challenges and responsibilities in caring for the most vulnerable during the COVID-19 pandemic. J Am Geriatr Soc 2020;68:1172-3.

13 McIntosh L. Can the COVID-19 crisis strengthen our treatment escalation planning and resuscitation decision making? Age Ageing 2020;49:525.

Address for correspondence: Dr Jatinder S Minhas, University of Leicester, Department of Cardiovascular Sciences, Infirmary Square, Leicester LE2 7LX, UK. Email:jm591@le.ac.uk 